\title{
RAFA Lens for Enhanced Far Focused Probes, Imaging and Analytical Resolutions
}

Muniyat Rafa and Rodney Herring

University of Victoria, Victoria, British Columbia, Canada

Since the beginning of optics whether focusing a beam by a refractive lens or reflective lens, the intensity at and close to the optic axis has not been able to be focused to a distant, tight, three-dimensional (3D) probe due to the absence of or shallow convergence angle afforded by beam. This limitation has resulted in the formation of an elongated, elliptical intensity profile along the optic axis with reduced intensity limiting the resolution of both 3D optical imaging and 3D analytical analyses of specimens in the direction along the optic axis of the microscope. These focusing problems have been solved with the RAFA lens (reflective advanced focusing aperture lens) accomplished using a 3D shaped cone reflector having a surface that is very slightly curved like a lens to focus a plane wave to the distant 3D spot (Figure 1). After the plane wave reflects off the surface of the cone reflector, it then reflects off of a flat mirror to form the high-intensity, far focused spot now able to be used as a probe for lasers and acoustic beams (Figure 2).

The reflective RAFA lens obeys Snell's law, which enables all wavelengths to be focused to the same focused probe position, and it works for both acoustic and laser beams. Multiple beams of different wavelengths can be simultaneously focused to the same position. Additionally, since the focused probe position lies on the optic axis and the focusing is strictly dependent on the cone's curved surface, the aberrations such as spherical aberration, chromatic aberration and coma can be fully corrected, enabling the size of the focused probe to be determined by the planarity of the beam and its associated wavelength. Shifting the cone reflector forward or backwards along with the optic axis shifts the focused probe position along the optic axis enabling limited focusing of the specimen during imaging and analytical analyses. Application of the RAFA lens to electron and ion beams is possible by applying a repulsive potential on its surfaces, i.e., the cone reflector and reflecting mirror. Increasing or decreasing the repulsive potential on the reflecting mirror will also shift the focused spot enabling movement of the beam on and off the optic axis. Off-axis circular motion of the beam can occur by using several electrostatic plates for the reflecting mirror, which is circular, having different charge potentials on each plate rotating 360 degrees.

Grants from NSERC Discovery, CFI and BCKDF are greatly appreciated.

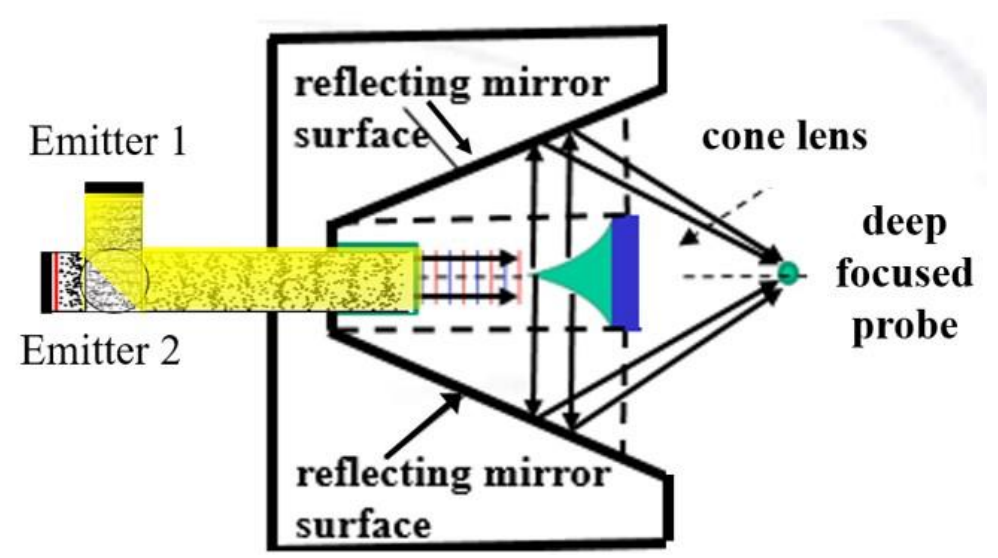


Figure 1. Configuration of the RAFA lens showing two plane wave emitters, each with full intensity focused to the same focusing position by reflecting off the cone lens and then off a reflecting mirror surface. The two emitters can have different wavelengths. An enhanced 3D intensity profile enables higher-resolution imaging and analytical analyses compared to the typical currently used lens design.
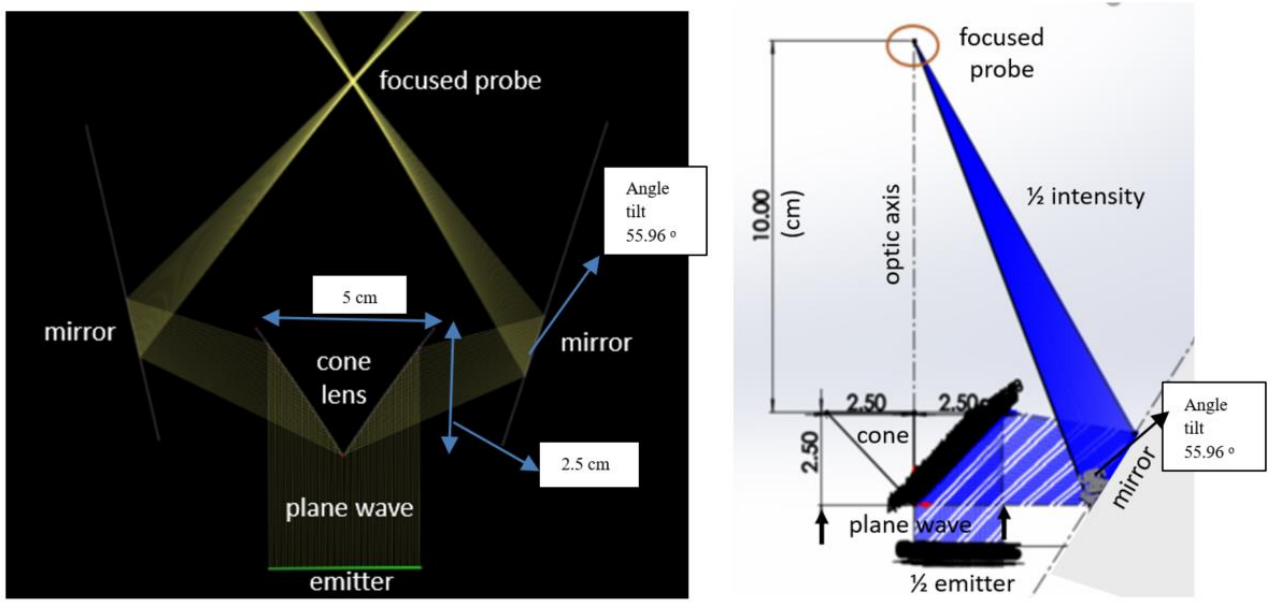

Figure 2. a) Ray path simulations showing the beam's intensity reflecting off the cone lens and the side mirror to form the far focused probe, b) a constructed RAFA lens for acoustic beams and lasers, and c) its visible demonstration focusing a HeNe red laser to a high-intensity, tight focused probe spot located $10 \mathrm{~cm}$ from the RAFA lens.
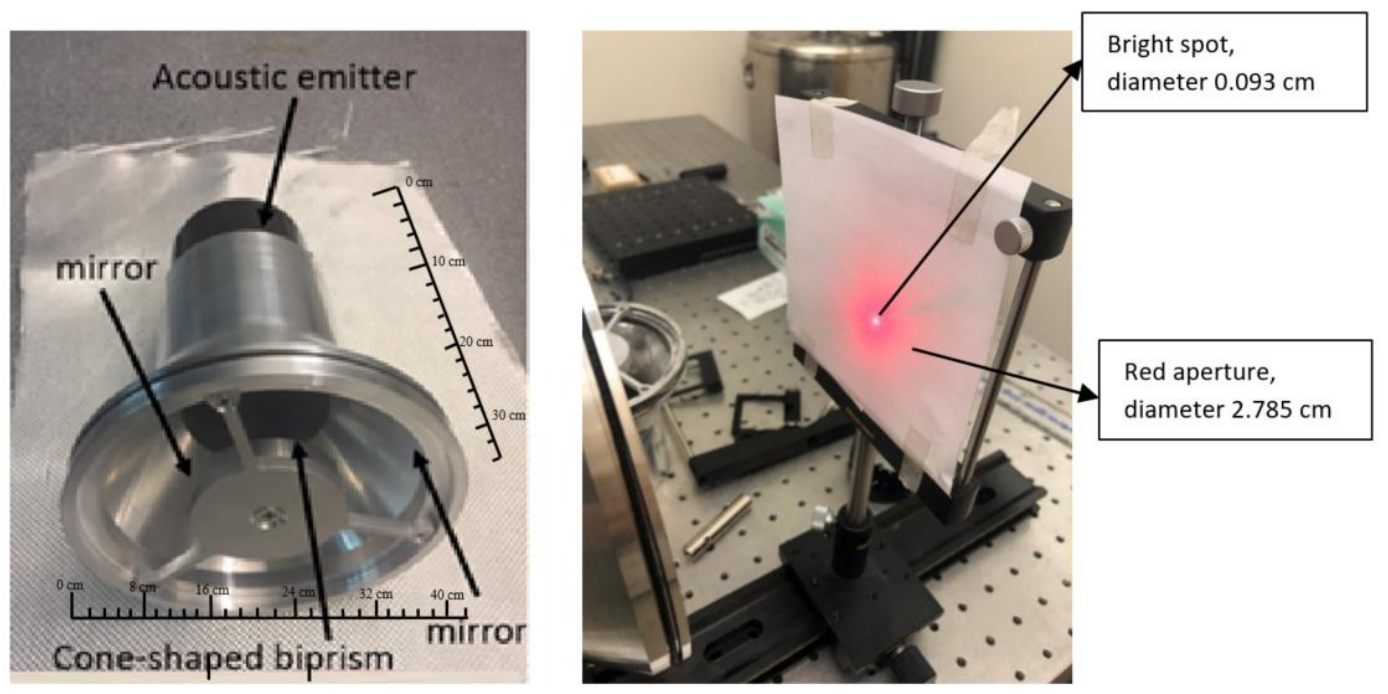

Figure 3. Left: CNC machined RAFA lens made of aluminum used with laser and acoustic beams. Right: The demonstration of the focusing properties using a He-Ne red laser; is shown the beam focused to a bright, highintensity spot, $0.93 \mathrm{~mm}$ diameter, located at a distance of $10 \mathrm{~cm}$ from the RAFA lens. 